IAC-06-B6.2.08

\title{
GENERALIZED ORBITAL PROJECTIONS OF A SUBLIMATING ICE PARTICLE
}

\author{
Evgeny Menkin, Ph.D., \\ ARES Corporation, Houston, TX USA \\ evgeny.menkin-1@nasa.gov \\ Jack Bacon Ph.D. P.E. \\ NASA Johnson Space Center, Houston TX USA \\ john.bacon-1@nasa.gov
}

\begin{abstract}
The issue of orbital debris resulting from human activities in space is a growing concern for the space users' community. Waste generated in Low Earth Orbit (LEO) can stay in orbit for a long time, creating significant hazards for other spacecraft flying at lower intercepting orbits. Many spacecraft, especially crewed vehicles, are required to vent fluids into space. These fluids include propellant, wastewater, excess condensate, and others. It is important to analyze the behavior of particles that result from these activities, since each individual particle is capable of damaging or destroying a spacecraft in a lower, crossing orbit, and such particles are invisible to tracking radar systems on the ground. The deorbit trajectory of an ice particle is complex. It depends on factors including attitude of the vehicle during vent, initial velocities of particles, altitude at which the vent occurred, and numerous evaporation and sublimation factors. These include contamination within the vented water, evolution of bubbles within the clear water, and sun flux factors such as time of the year and current beta angle.

The purpose of this study is to examine the influences of these factors on the trajectories of ice particles resulting from condensate water dumps, and to bound the safe trajectories of spacecraft that lie below the venting spacecraft.
\end{abstract}

\begin{tabular}{|c|c|c|c|c|}
\hline & & $\begin{array}{l}\text { NOMENCLATURE } \\
\text { (in alphabetical order) }\end{array}$ & & \\
\hline$A$ & $=$ surface area of the particle & $I_{\text {therm }}$ & $=$ & thermalization coefficient \\
\hline Alt & $=$ altitude of the particle & $K$ & $=$ & empirical constant \\
\hline$B_{N}$ & $=$ ballistic number & $k$ & $=$ & Boltzmann constant \\
\hline$C_{D}$ & $=$ coefficient of drag & $k_{1}$ & $=$ & sublimation rate constant \\
\hline$C_{p}$ & $=$ ice heat capacity & $L$ & $=$ & heat of sublimation \\
\hline$c_{0}, c_{g}, c_{0}^{\prime}$ & $=$ surfactant concentrations & $M$ & & $\begin{array}{l}\text { molecular weight of diffusing } \\
\text { substance }\end{array}$ \\
\hline$D$ & $=$ diffusion coefficient & $m$ & $=$ & water molecule \\
\hline
\end{tabular}




$$
\begin{aligned}
& m_{c}=\text { complex index of refraction } \\
& N=\text { GMT day number } \\
& n, i k=\text { real and imaginary indexes of } \\
& \text { refraction respectively } \\
& P(T)=\text { vapor pressure above the particle } \\
& p_{b} \quad=\quad \text { internal pressure of the bubble } \\
& p_{s} \quad=\text { pressure at the particle surface } \\
& p_{v}\left(T_{0}\right)=\text { internal vapor pressure at } T_{0} \\
& Q_{\text {Albedo }}=\text { reflected heat flux by Earth } \\
& Q_{\text {atm.heat }}=\text { collisional heat flux } \\
& Q_{\text {Earth }}=\text { heat flux from the Earth } \\
& Q_{\text {in }} \quad=\text { incoming heat flux } \\
& Q_{\text {Space }}=\text { heat flux emitted to space } \\
& Q_{\text {Sunaverage }}=\text { average heat flux from the Sun } \\
& Q_{\text {Sunreal }}=\text { real heat flux from the Sun } \\
& R \quad=\text { gas constant } \\
& R_{b} \quad=\quad \text { radius of the bubble } \\
& R_{0}, R_{\text {drop }}=\text { radius of the particle } \\
& R_{\text {Earth }}=\text { Earth radius } \\
& T_{\text {drop }}=\text { particle temperature } \\
& T_{\text {Space }}=\text { space background temperature } \\
& V \quad=\text { velocity of the particle } \\
& \frac{d R}{d \tau} \quad=\quad \text { rate of sublimation } \\
& \alpha(T)=\text { molecular sticking fraction } \\
& \chi\left(R_{\text {drop }}, \lambda\right)=\text { absorption coefficient } \\
& \varepsilon=\text { emissivity coefficient } \\
& \varphi_{\text {Earth }}=\text { Earth viewfactor } \\
& \varphi_{\text {Space }}=\text { Space viewfactor } \\
& \lambda=\text { wavelength } \\
& \rho_{\text {atm }}=\text { atmospheric density } \\
& \rho_{\text {drop }}=\text { ice density } \\
& \sigma \quad=\text { surface tension } \\
& \sigma_{B}=\text { Stefan - Boltzmann constant } \\
& \tau \quad=\text { time }
\end{aligned}
$$

\section{INTRODUCTION}

When a vented stream starts disintegrating due to rapid expansion of dissolved gasses, it creates many millions of particles of different size and shape. The outer layers of such particles are cooled by fast evaporation from the surface. Larger particles tend to continue to break up until surface tension equilibrates expansion forces that otherwise tear particles apart. Previous studies predict particle sizes to be comparable to the initial diameter of the stream [1] which is up to twice the size of the venting nozzle. For this study we determined that droplets will completely freeze within 10 seconds after the vent with average diameter of $2 \mathrm{~mm}$. Although there is a large number of misted particles in addition to the main stream, this particular study cannot be used for the particles of very small diameters, where Mie scattering is applied. We did not perform sublimation analysis of such small particles because the intent of this study was to characterize only the particles that posed a ballistic threat. Analysis starts at the time when a droplet is completely frozen and continues until the droplet reaches an altitude of 250 kilometers, or sublimates to one hundredth of its original size. Once formed, droplets cool very quickly and reach equilibrium temperature of $\sim 190 \mathrm{~K}$ in the order of seconds. This study looks at different aspects that affect (A) the sublimation rate, such as: beta angles, time of the year, initial altitude, impurities, surfactants, etc. and (B) the deorbit rate, such as: the initial altitude at which vent has occurred, numerous atmospheric parameters and the drop/particle cross section, which is completely determined by (A) above.

\section{ASSUMPTIONS}

In order to simplify our study, we made several assumptions to our model. We will address the rationale and the effect of the assumptions later in the paper. For the modeling purposes we assumed a spherical crystalline clear ice particle that has no impurities or organic solvents. Particles were assumed to have a clear surface (no roughening) and to be homogeneous (have no dissolved bubbles). The background temperature for Earth was taken to be $255 \mathrm{~K}$ at $50 \mathrm{~km}$ reference altitude and background temperature of space was assumed to be $3 \mathrm{~K}$. The absorptivity of Earth IR by the ice particle was calculated from complex indices of refraction to be approximately $\chi(R, \lambda)=0.93$. In the atmospheric model we assumed that after venting, the particles remain in circular or nearly circular orbit with initial venting altitude of $400 \mathrm{~km}$. The coefficient of drag $C_{D}$ was assumed to be 2 , characteristic of very smooth spherical particles.

\section{MODELING}

In vacuum large diameter streams with high vapor pressures have a tendency to burst, forming a cloud of particles which subsequently freeze and eventually sublimate. The behavior of the stream 
and the formation of the particles in vacuum depend on several factors such as vapor pressure, viscosity of the vented fluid, dissolved gas content, surface tension, surfactants and the density.

\section{PARTICLE FORMATION}

"Boiling" of dissolved gasses and surface heat transfer due to rapid evaporation are governed by a diffusion equation [2]. It can be assumed by basic transport considerations that rapid changes in a liquid's temperature or dissolved gas content occur in a relatively thin layer near the surface, and it will freeze from the outside in, trapping most dissolved gasses inside the drop. However, we do not expect gasses to evolve into bubbles. It is obvious that for a bubble to exist or to grow its internal pressure has to be at least greater than the competing pressures of the drop and bubble wall curvatures:

$p_{b}=p_{s}+\frac{\sigma}{R_{\text {drop }}}+\frac{2 \sigma}{R_{b}}$,

Assuming small thermal gradients in the small particle, the internal bubble pressure must be at the vapor pressure $p_{v}\left(T_{0}\right)$ and $p_{s} \approx 0$ for a very cold surface.

Thus, a minimum bubble radius that permits growth is

$R_{b}=2 \sigma\left(p_{v}-\frac{\sigma}{R_{\text {drop }}}\right)^{-1}$,

for such a cold particle surface. Figure 1 schematically shows bubble growth in the particle.

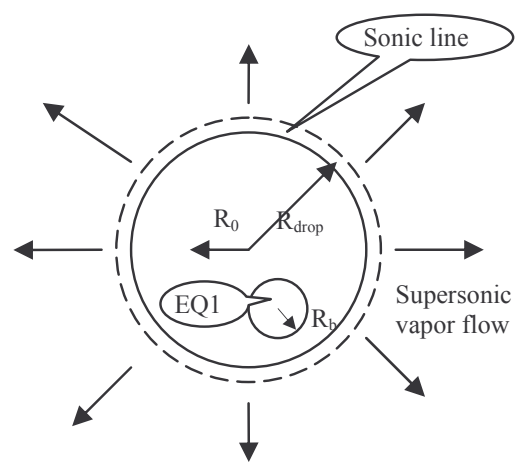

Figure 1 Bubble expansion in the particle

From equation (2) we can calculate that for cavitations to occur in $2 \mathrm{~mm}$ diameter particles the radius of the bubble $R_{b}$ has to be greater than $10 \%$ of the radius of the particle, or larger for smaller particles. Thus there is a lower potential for particles to contain bubbles as initial drop size decreases. Any pre-existing bubbles will act as fracture points in the original stream, not tending to remain encapsulated in the liquid.

As a particle freezes the expansion of water will apply increasing internal pressure to the inner portions of the particle as the thickness of the ice layer increases [3]. This, combined with the facts that particles will be rotating, have high surface tension, and quickly rising internal pressure, allows us to assume a spherical shape for the particle.

\section{ENVIRONMENT MODEL}

The energy budget for liquid or solid water particles in LEO consists of four major components: heat flux from the Sun plus Sun flux re-radiated from Earth atmosphere, infra-red heat flux from the Earth, blackbody radiation to deep space, and sublimation. Heating due to radiation from Earth's lower atmosphere is extremely small, because water vapor in the atmosphere absorbs pretty much in the same spectral regions as solid or liquid water particles in the orbit. The re-radiated spectrum form higher in the atmosphere has the most effect on water drops or ice particles in orbit. Heating from molecular collisions is easily shown to be negligible at orbital altitudes and thus is not accounted for in this study. Using above mentioned assumptions, the energy balance for the particle can be represented by the following formula:

$$
\begin{aligned}
& \frac{4}{3} \pi R_{\text {drop }}^{3} \rho C_{p} \frac{d T_{\text {drop }}}{d \tau}= \\
& =\sum Q_{\text {in }}-4 \pi R_{\text {drop }}^{2} \rho L \frac{d R}{d \tau}-, \\
& \pi R_{\text {drop }}^{2} \sigma_{B} \varepsilon\left(T_{\text {drop }}^{4}-T_{\text {Space }}^{4}\right)
\end{aligned}
$$

where

$$
\begin{aligned}
& \sum Q_{\text {in }}=\chi\left(R_{\text {drop }}, \lambda\right) Q_{\text {Surreal }}+Q_{\text {Earth }}+ \\
& +\chi\left(R_{\text {drop }}, \lambda\right) Q_{\text {Albedo }}+Q_{\text {atm.heat }}
\end{aligned} .
$$

\section{LONG WAVE RADIATION ABSORPTION}

Water droplets and ice particles have very similar absorption characteristics with more intense absorption in the long wave region. It was shown by Kondratyev et all [4] that in the Earth infra-red spectral region, the main portion of which is localized at wavelengths from 4 to $40 \mu$, almost all energy is completely absorbed by the atmosphere and re-radiated, leaving two transparency 
windows in 8 to $12 \mu$ and 16 to $24 \mu$. We have calculated from the energy spectrum and real and imaginary indices of refraction over that range that $7 \%$ of the flux is reflected from a crystalline ice particle. For our model we calculated particle absorptivity to be $\chi\left(R_{d r o p}, \lambda\right)=0.93$ of Earth IR.

For modeling purposes and in order to account for the spectral intensity of the scattered infra red, Earth is assumed to be a blackbody with a lower than usually assumed temperature at $255 \mathrm{~K}$. Figure 2 shows black body emission curves for Sun and Earth.

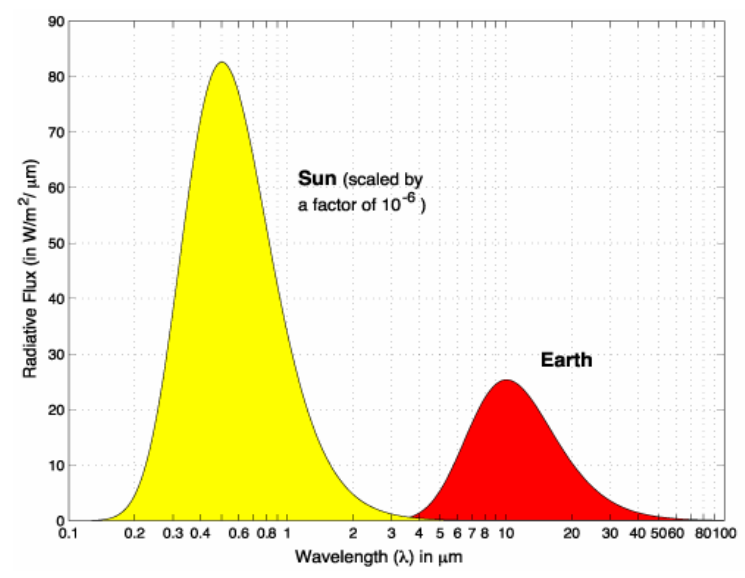

Figure 2 Black Body Emission Curves of the Sun and Earth

The view factor is calculated for the altitude of the particle as it rapidly deorbits:

$\varphi_{\text {Earth }}=\frac{1-\sqrt{1-\left(\frac{R_{\text {Earth }}+50}{R_{\text {Earth }}+\text { Alt }}\right)^{2}}}{2}$,

where $50 \mathrm{~km}$ was chosen to be a reference (equilibrium) altitude for a black body temperature of $255 \mathrm{~K}$, where IR spectrum is constantly absorbed and reemitted below this altitude.

By calculating the Earth view factor we can calculate long wave radiation received from Earth at a specific altitude:

$$
\begin{aligned}
& Q_{\text {Earth }}= \\
& 4 \pi \chi\left(R_{\text {drop }}, \lambda\right) \sigma_{B} R_{\text {drop }}^{2}\left(T_{\text {drop }}^{4}-T_{\text {Earth }}^{4}\right) \varphi_{\text {Earth }},
\end{aligned}
$$

\section{SUNLIGHT ABSORPTION}

For particles larger than 100 microns in diameter, both $Q_{\text {Albedo }}$ and $Q_{\text {sunaverage }}$ are calculated using geometric optics, analyzing the path length of each wavelength within the sphere as a multiple of its absorption length.
The total energy flux from the Sun, with a blackbody spectrum of $6000 \mathrm{~K}$, averages to 1363 Watts $/ \mathrm{m}^{2}$ in Near Earth Orbit (NEO).

Daily variations due to the eccentricity of Earth's orbit can be calculated as:

$$
\begin{aligned}
& Q_{\text {Sunreal }}= \\
& Q_{\text {Sunaverage }}\left(1+0.034 \cos \left(\frac{360 N}{365.25}\right),\right.
\end{aligned}
$$

Although yearly variations of solar radiation are within $5 \%$ of average, they were easy to account for and thus were used in the model. These variations may play a bigger role in the future modeling, when the effects of surfactants, impurities and of surface roughness are considered, causing absorption of solar flux to be a more dominant fraction of the heat balance [5].

Below 100 micron diameter, in optics it is important to consider the effects of Mie scattering. This phenomenon causes an increasing portion of the light to be scattered as the drop radius approaches the comparable wavelengths. Thus, the heat input slows, and the sublimation rate rapidly falls off.

Interesting Hypothesis: Droplets smaller than 100 microns are not considered in this study. However there is a potential that due to vanishingly small sublimation rates and infinitesimal ballistic number, they may survive atmospheric impact and seed the highest reaches of the atmosphere as tiny pure ice crystals. Orbital speeds and atmospheric densities can decelerate a sub-micron particle in seconds to thermal speeds. This phenomenon, if verified, may partially explain the recent proliferation of noctilucent clouds, which were extremely rare before this century and which are becoming increasingly common in the space age. Noctilucent clouds appear at $80-100 \mathrm{~km}$ altitudes above Earth, and reflect sunlight long after dusk and long before sunrise. Such clouds need both concentrations of water vapor at high altitudes (reasonably common) and a large number of seed particles at $80-100 \mathrm{~km}$ altitude (unexplained and previously quite rare, occurring usually only after massive volcanoes). The tightly clustered de-orbit of billions of sub-micron particles in 
spacecraft water vent operations may seed such clouds.

Numerous attempts have been made to simplify the absorption process of sunlight in small droplets as a simple fraction of incident flux. Unfortunately, there are many nonlinear effects that drive this process, which must be integrated over all wavelengths over the entire illuminated surface in a range of droplet sizes. Ice exhibits a different real and imaginary index of refraction for wavelength in the solar spectrum. The combination of these indices determines the amount of reflection (which varies with polarization, but is always averaged 50:50 between vertical and horizontal polarization over a sphere), the extent of refraction, and the fraction of energy in the refracted portion that is ultimately absorbed [6]. The absorption coefficient can be written in a form of the equation:

$$
\chi\left(R_{\text {drop }}, \lambda\right)=\operatorname{Re}\left\{\frac{i 8 \pi R_{\text {drop }}\left(m_{c}^{2}-1\right)}{\lambda\left(m_{c}^{2}+2\right)}\right\},
$$

where

$m=n-i k$

( $m$ is a complex index of refraction).

The real and imaginary indexes of refraction vary greatly by wavelength as shown in Figure 3.

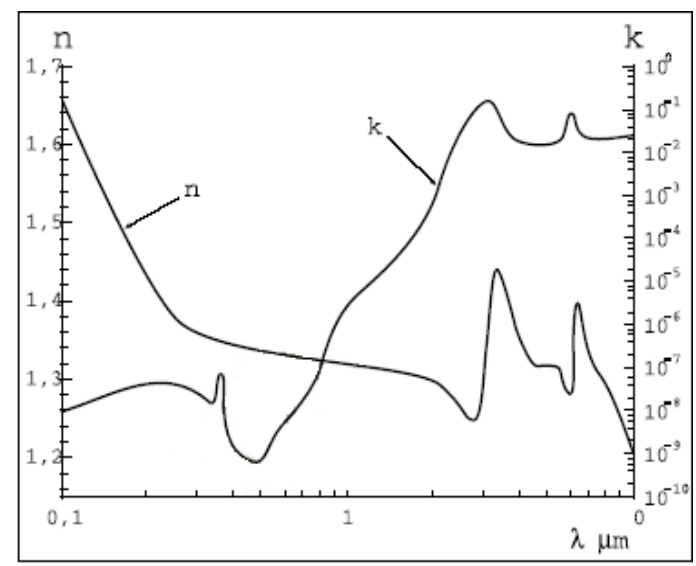

Figure 3 Real and imaginary indexes

Our study shows that heat flux from the Sun $Q_{\text {sun }}$ absorbed by a crystalline spherical ice particle (with no impurities or bubbles) is negligible in comparison to other heat fluxes affecting the particle. For the studied range of particle sizes, the absorbed solar energy varied from 25 to $45 \mathrm{~W} / \mathrm{m}^{2}$, which is only $1.8 \%$ to $3.3 \%$ of the incident solar flux, and a small fraction of the absorbed infra red from the Earth. The calculated values of absorbed fraction of solar flux versus drop size are presented in Figure 4, along with simplified empirical fit that was useful in computer calculations of ballistics of particles $<2.5 \mathrm{~mm}$ radius. As the particle becomes smaller, the fraction of the sunlight absorbed gets smaller, due to decreasing path length for absorption of the different spectra. This effect is not seen in Earth IR absorption, due to the very short absorption length of IR in water relative to the smallest drop diameters.

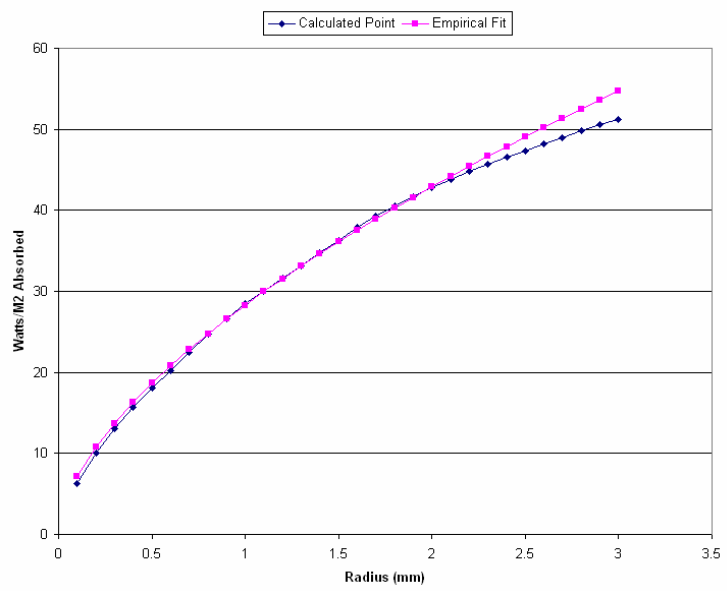

Figure 4 Sun absorption in crystalline sphere

\section{EARTH ALBEDO}

The term albedo (Latin for white) is commonly used to describe the overall average reflection coefficient of an object. The Earth's albedo is composed of sunlight reflected from the surface of the planet and scattered from the top layers of the atmosphere. Heat flux from reflected sunlight $Q_{\text {Albedo }}$ is absorbed by the crystalline clear spherical ice particle the same way as direct sunlight $Q_{\text {sunreal }}$. The difference between the absorption of Earth albedo and of direct sunlight is in the view factor of the sphere, and the incident intensity. Since the ice particle moves at very high velocity in the LEO and environment changes very fast we can use the average Earth albedo of 0.3 throughout the illuminated part of the orbit.

Heat flux reflected form the Earth can be calculated using the following formula:

$$
Q_{\text {Albedo }}=0.3 \chi\left(R_{\text {drop }}, \lambda\right) Q_{\text {Sunreal }}
$$

\section{BALLISTIC COLLISIONS}

The energy added to the particle from direct elastic collision with a rarified gas can be only a fraction of the incident energy flux. 


$$
Q_{\text {atm.heat }}=I_{\text {therm }} \frac{\pi R_{\text {drop }}^{2} \rho_{\text {atm }} V^{3}}{2},
$$

Since atmospheric densities at ISS altitudes are on the order of $10^{-11}$ to $10^{-12} \mathrm{~kg} / \mathrm{m}^{3}$ and velocities are less than $8 \mathrm{~km} / \mathrm{sec}$, the maximum energy deposited to the particle cannot be greater then 6 Watt $/ \mathrm{m}^{2}$, even assuming that all energy was thermalized, which is not true. Most collisional energy is imparted in the form of deceleration. Thus for the modeling purposes $Q_{\text {ballistic }}$ is negligible compared to other energy fluxes, and is assumed to be equal to zero.

\section{ENERGY LOSS}

\section{RADIATION TO SPACE}

The view factor to space is calculated as:

$\varphi_{\text {Space }}=1-\varphi_{\text {Earth }}$

then long wave radiation emitted to space can be calculated as

$$
Q_{\text {Space }}=4 \pi \varepsilon \sigma_{B} R_{\text {drop }}^{2}\left(T_{\text {drop }}^{4}-T_{\text {Space }}^{4}\right) \varphi_{\text {Space }},
$$

\section{SUBLIMATION}

In the process of formation particles will rapidly start loosing their energy through the process of evaporation and then, when they subsequently freeze, through the process of sublimation. This sublimation becomes the principal mechanism of energy loss. Sublimation is the process whereby a solid substance changes its state skipping a phase, going from solid to vapor. This is the only possible path for ice below $0.006 \mathrm{~atm}$, such as the particle experiences in space. Figure 5 shows triple point chart for water [7].

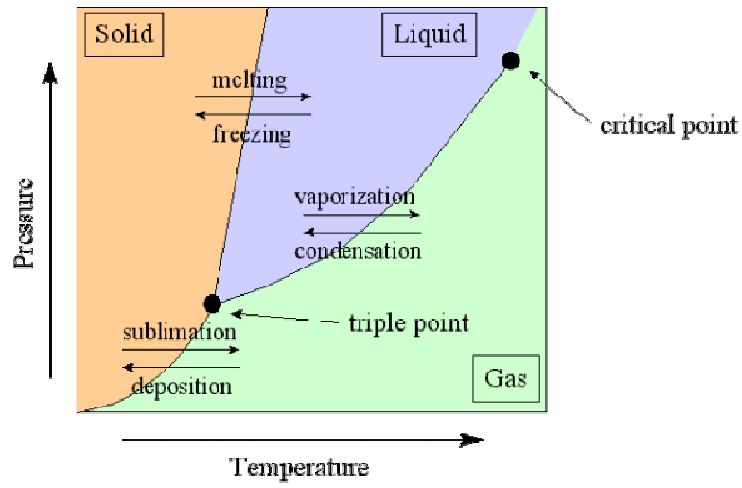

Figure 5 Triple Point Chart

The initial temperature of the drop does not affect the final equilibrium temperature. The drop reaches its freezing point in seconds and its equilibrium temperature in minutes if external heat fluxes do not change.

The heat of sublimation can be calculated using the following formula:

$Q_{\text {sub }}=L A \frac{d R}{d \tau}$,

The rate of sublimation

$\frac{d R}{d \tau}(T)=\alpha(T) P(T) \sqrt{\frac{m}{2 \pi k T}}$,

depends on the equilibrium vapor pressure of liquid and solid water. Sublimation rates per unit exposed area are proportional to this pressure and temperature of the drop $[8,9]$.

Equilibrium pressure for pure ice is

$P(T) \approx 3.2 e 10^{12} \exp \left[-\frac{6110}{T}\right] P a$

\section{PARTICLE DECAY RATE}

Once particles are formed they will start slowing down linearly proportional to their $\frac{\text { Area }}{\text { mass }}$ ratio.

We took $C_{D}=2$, since we assumed particles to have spherical form. While decaying, particles will sublimate, constantly changing their ballistic number which will expedite particle decay rate.

$\frac{d V}{d \tau}=\frac{1}{2} C_{D} \frac{A}{m} \rho_{A t m} V^{2}$

Where $\frac{A}{m}$ is the ratio of area of the ice particle to the mass of the ice particle:

$\frac{A}{m}=\frac{\pi R_{\text {drop }}^{2}}{\frac{4}{3} \rho_{\text {drop }} \pi R_{\text {drop }}^{3}}=\frac{3}{4} \frac{1}{\rho_{\text {drop }} R_{\text {drop }}}$

If we integrate sublimation rate of the drop over one orbit period we will see later that we can approximate $R_{\text {drop }}(\tau)$ to linearly decrease with time:

$R_{\text {drop }}(\tau)=R_{o}-k_{1} \tau$

where $R_{o}$ is the initial ice particle diameter, and $k_{l}$ being a very small constant sublimation constant, on the order of nanometers per second.

The change in altitude with time is differentially linked to the change in velocity over time. Since: 


$$
\begin{aligned}
& V=\sqrt{\frac{\mu}{\left(R_{\text {Earth }}+\text { Alt }\right)}} \\
& \frac{d\left(\text { Alt }+R_{\text {Earth }}\right)}{\left(\text { Alt }+R_{\text {Earth }}\right)}=2 \frac{d V}{V}
\end{aligned}
$$

Combining equations $18,19,21$, and 22 , we can get the equation:

$$
\frac{d A l t}{d \tau}=\frac{3}{2} \frac{C_{D}}{\rho_{\text {drop }} R_{\text {drop }}} \sqrt{\frac{\rho_{\text {Atm }}(\mu)}{\left(\text { Alt }+R_{\text {Earth }}\right)}}
$$

Where Alt, $R_{\text {drop }}$ and $\rho_{\text {Atm }}$ are all varying with time. We have chosen to close the model with digital-numeric integration of equation (23), due to the variation of density and of sublimation rate with time and other nonlinearities. However, in the case of crystalline clear spherical particle it is possible to get a workable analytic solution for particle deorbit using equation (20) and assuming a time-invariant density profile that is purely a function of altitude. Then the solution can be closed by rearranging equation (23) to make one side completely dependent upon time, and the other side completely dependent upon altitude:

$$
\begin{aligned}
& \int_{0}^{t} \frac{d \tau}{\left(R_{o}-k_{1} \tau\right)}= \\
& \frac{2}{3} \frac{\rho_{\text {drop }} \sqrt{\mu}}{C_{D}} \int_{\text {Alt }(0)}^{\text {Alt }(t)} \sqrt{\left(A l t+R_{\text {Earth }}\right)} \rho_{\text {atm }}(\text { Alt }) d(\text { Alt })
\end{aligned}
$$

The left-hand side is a simple logarithm in time $\tau$, scaled by the constant sublimation rate $k_{l}$ and $R_{o}$, the initial size of the drop. The initial radius of the particle $R_{o}$, and sublimation rate $k_{l}$ are the driving factors for the particle time in orbit. Exponentiation of both sides leads to a scaled time $\tau$ being a complicated, characteristic function of $\operatorname{Alt}(t)$ and initial release altitude $\operatorname{Alt}(0)$. However, the right-hand side typically does not close analytically because of the mixture of powers and exponentials in altitude under the integral. It can be approximated to arbitrary precision by repetition if the techniques of integration of the product of functions. We can do similar tricks to determine the phase angle of the particles ahead of the releasing spacecraft. This is essential when predicting the time and altitude of orbit plane crossings with spacecraft in lower orbits. Ultimately, the limitations imposed on the precision of our analysis by the need to assume overly simplistic atmosphere density and sublimation rate, and the ability to only approximate the integral, led us to develop full numeric integration tools to solve this problem.
Our analysis has shown that atmospheric density variation has the most impact on particle ballistics. Atmospheric modeling is therefore most critical to a precise ballistic forecast especially for orbital objects with infinitesimally small $\mathrm{B}_{\mathrm{N}}$. For a simple exponential function of density vs. altitude and slightly-varying reference scale height, depending upon which F10.7 number was chosen, the decay varies from $\sim 17$ hours to $\sim 54$ hours to decay from 400 to $250 \mathrm{~km}$. This dependence is shown on Figure 6.

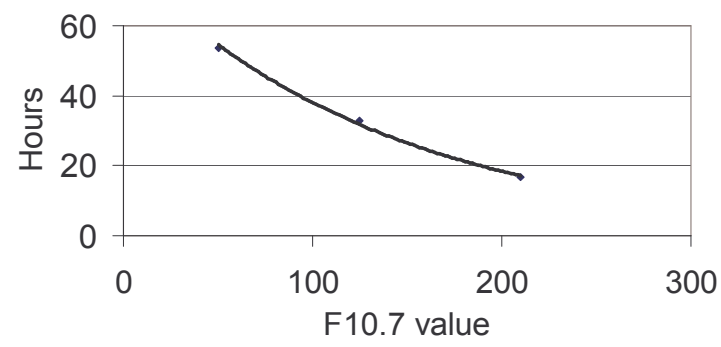

Figure 6 Atmospheric density effect on decay rate

Because of the sensitivity to density, we have explored several different models of the atmosphere to process the ballistic forecast. Generally, all sophisticated models require precise inputs of location, altitude, F(10.7) (both averaged over three months, and daily values), geomagnetic potential, and beta angle. We must accept our largest uncertainty in the forecast from the atmospheric modeling, because the most accurate atmospheric and ballistic models often take fixed scalar inputs, which do not work in our case because of rapidly changing $B_{N}$ and altitude decay rate. We are in the process of building a simplified model of the atmosphere specifically for this kind of work as well as developing plans for fully integrated ballistic tool that incorporates real time atmospheric density and sublimation variations.

\section{ANALYSIS}

As a part of our water vent study we developed a small tool that calculates decay rate for the sublimating crystalline clear spherical ice particle, incorporating many small, nonlinear effects which are ultimately more important for contaminated water or for other effluents. We did integration using simple Runge-Kutta method simultaneously solving for three equations: temperature $\frac{d T}{d \tau}$, radius $\frac{d R}{d \tau}$ and altitude $\frac{d A l t}{d \tau}$ change.

We can point out three major phases in sublimation of deorbiting ice particle. In the initial 
phase the temperature and the radius of the particle change relatively rapidly as the equilibrium is established.

Our analysis begins a few seconds after the water is vented. We assume that particles will cool down to $273 \mathrm{~K}$ within approximately 6 to 8 seconds after the vent and form $2 \mathrm{~mm}$ diameter crystalline clear spheres. Within first 30 seconds of our calculation, the particle cools down to $\sim 200$ $\mathrm{K}$ shrinking almost $1 \%$ from its original size. Figures 8 and 9 show the temperature drop rate and radius loss rate respectively.

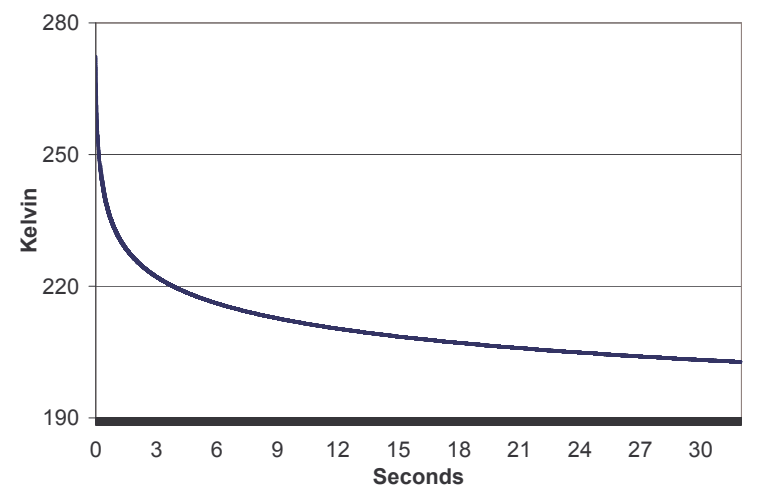

Figure 8 Particle initial temperature change rate

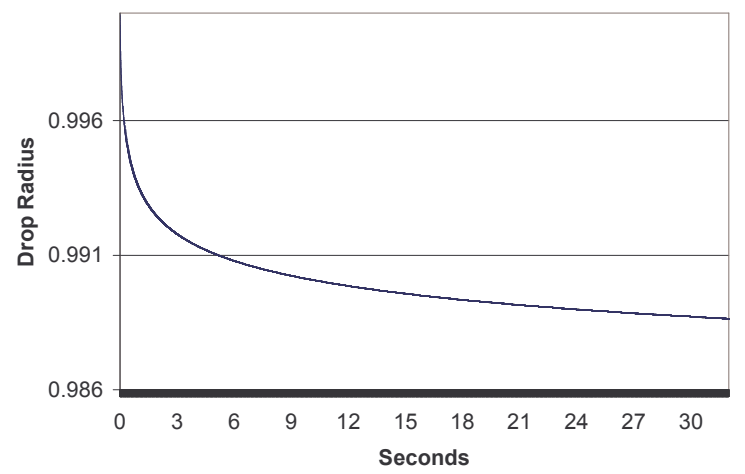

Figure 9 Particle initial radius change

The particle experiences tremendous energy loss during first 30 seconds primarily due to the sublimation process as the equilibrium temperature is reached. This expels approximately $2 \%$ of particle's original mass and volume.

From the very first moment particle starts rapidly descending and looses $\sim 10$ meters of altitude in the first 30 seconds.

After that, there is a long phase where the radial reduction rate is nearly constant with time, followed by a third final phase, when competing influences may make the rate change in the last stages.
Following the establishment of thermal equilibrium, the rate of radial reduction of the particle is essentially constant. The radiated energy fluxes are all proportionate to the surface area, where the sublimation rate is proportional to the surface area times $\frac{d R}{d \tau}$. Thus, $\frac{d R}{d \tau}$ will remain nearly constant, while environment remains constant. In the very latest stages of orbital decay the variations in flux rate due to changing view factors will have larger effect. Figures 10 and 11 show changes in temperature and particle size.

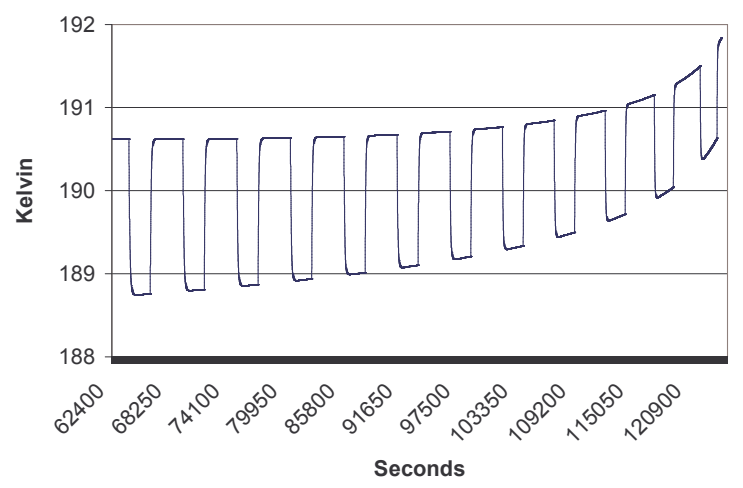

Figure 10 Particle temperature change

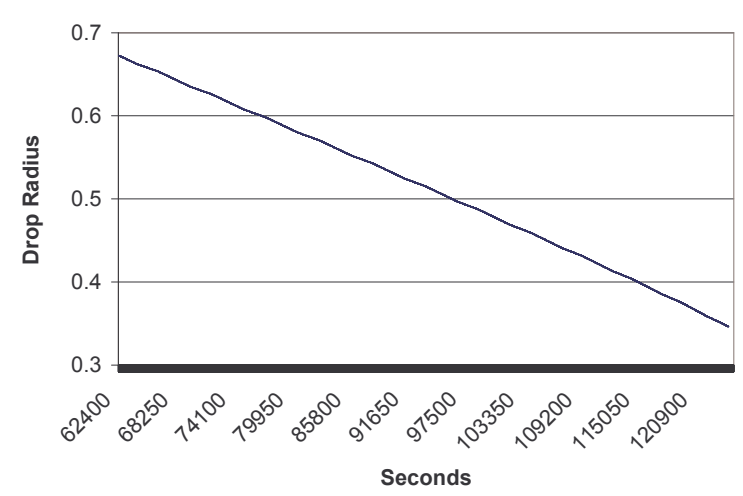

Figure 11 Particle mass loss

In later stages, although Earth view factor rapidly increases, as the droplet shrinks, sunlight wavelengths are even less absorbed. This is due to the increasing effect of Mie scattering. Thus, competing influences try to increase the IR flux rate (from Earth view factor) and to reduce total flux rate (from reduced absorption length, and Mie scattering). The rate of surface ablation changes proportionally to change of the viewfactor. When particle approaches $\sim 320 \mathrm{~km}$ altitude the rate of orbital decay rapidly increases which leads to proportionate increase in sublimation rates and particle temperature increase. These dependencies are shown Figures 12 and 13. 


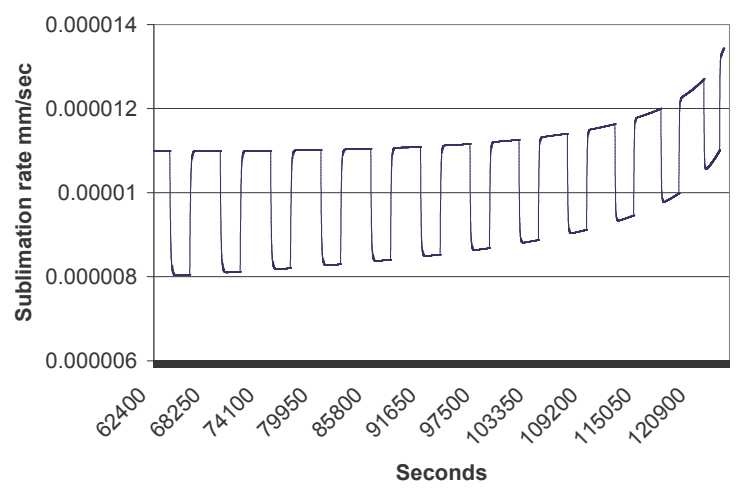

Figure 12 Particle sublimation rate

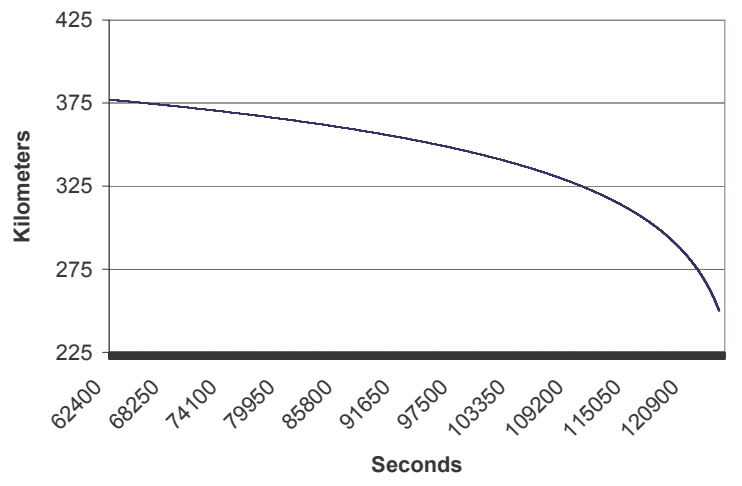

Figure 13 Particle decay rate

The process of sublimation continues until the particle size becomes comparable to wavelength of incoming radiation, where it drastically slows down or may even completely stop because particle scatters nearly all external radiation. Our study has shown that in the worst case scenario of low atmospheric density (F10.7 $\rightarrow 50)$ and cold environment (GMT day 173 and $0^{0} \beta$ angle) particles can survive in LEO no longer then 59 hours.

Analysis was performed for $0^{\circ}$ and $75^{\circ} \beta$ angles as well as for aphelion and perihelion conditions. Using constant atmospheric parameters we see that annual solar variations change particle life in orbit only by $0.6 \%$ and maximum $\beta$ angle variation (duration of eclipse) changes particle life by $8.1 \%$. These variations do not play significant role in determining particle life, compared to uncertainty in atmospheric density and in the range of particle sizes in the vented stream.

It is clear that the smaller particle gets, the less its temperature deviates from equilibrium temperature.

The atmospheric properties on the day of venting are crucial to the ballistics of these very light particles. Generically, the rate of decay increases as the particle gets smaller, altitude drops, and atmospheric density increases. From $400 \mathrm{~km}$ down to $250 \mathrm{~km}$ atmospheric density increases by almost two orders of magnitude. The change in atmospheric density using F10.7 number from minimal 50 to maximum 210 reduces particle life in orbit by $318 \%$, and the best possible model of the atmosphere is therefore imperative. As mentioned before, no current high fidelity atmosphere model used for ballistic predictions can incorporate such rapidly changing properties and altitudes as our problem engenders, and we are in the process of building new customized models to address this problem.

\section{SURFACTANTS}

The term "surfactant" is a blend of "Surface active agent". Typically surfactants are organic compounds that contain both hydrophobic groups and hydrophilic groups, although almost any contaminant has an effect. They are typically soluble in both organic solvents and water.

There is a lot of discussion in the literature on the effects of organic hydrocarbons on water evaporation and the ability of them to form monolayers that can significantly reduce evaporation. Water drop evaporation retardation by monolayers is possible because of their ability to assume more closely packed molecular orientation as the drop evaporates and the surface area contracts. Our assumption is that similar process will take place in space when a particle starts sublimating. We believe that it is possible for accumulating surface impurities to form a layer or coating on the surface of the liquid drop during the phase of ice particle formation and reduce the rate of sublimation $[10,11,12]$. The schematic of boundary layer formation is shown on Figure 14.

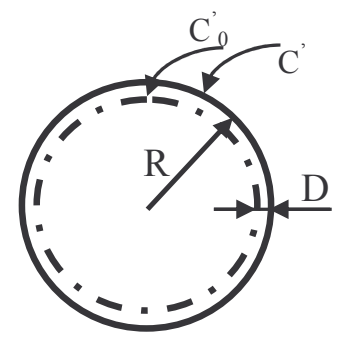

\section{Figure 14 Surface concentration}

The rate of evaporation of contaminated particle can be represented by the following formula:

$$
\frac{d R}{d \tau}=\frac{D}{R_{d r o p}}\left(c_{0}-c_{g}\right)
$$

As discussed above, the evaporation rate should remain constant if the external environment does not change. However the rate of evaporation 
reduces as the concentration of surfactants at the outer layer of the particle increases. Figure 15 shows the dependence of surfactants of evaporation rates.

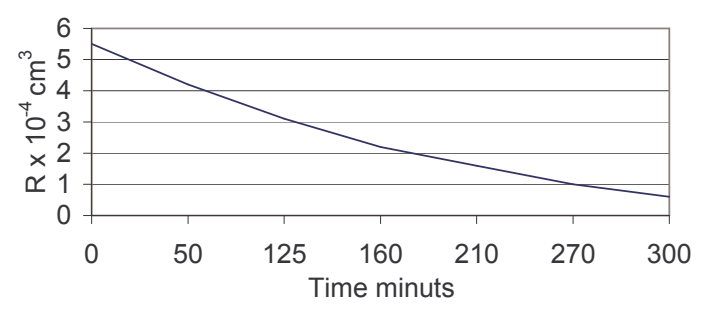

Figure 15 Evaporation rate reduction

The curvature of the drop does not play a large role in changing evaporation rate unless particles get too small, and since we are not concerned about very small particles we can neglect this effect:

$\ln \frac{c_{0}^{\prime}}{c_{0}}=\frac{2 \sigma M}{R_{\text {drop }} R T \rho}$

The rate of evaporation of the particle containing surface impurities can be represented as:

$$
\frac{d R_{\text {drop }}}{d \tau}=\frac{D}{R_{\text {drop }}} \frac{\left(c_{0}-c_{g}\right)}{1+\frac{K R_{0}^{2}}{R_{d r o p}^{3}}},
$$

where $K$ is found empirically.

It is still uncertain how fast the outer layer is formed and if it is carried away by initial process of particle formation. If it is formed within the time it takes for the particle to freeze, it is possible that the monolayer might be expelled in a kind of purifying step, in the violent evaporation phase. If not, it may remain as an inhibiting seal on the surface of the particle.

\section{WASTE WATER CONTAMINATION}

Currently there are two manned space vehicles designed to release waste water into space. The International Space Station (ISS) and Space Shuttle are each capable of releasing $\sim 60$ liters of waste water at one vent. The size of the cloud and the process of particle formation are similar for both vehicles. The major difference between the two is the duration of particle survival in LEO due to different impurities contained in vented water.

We have conducted a study of ISS waste water and determined that it contains mechanical impurities that differ in size from 25 to 400 microns. Figure 16 shows size distribution of impurities.

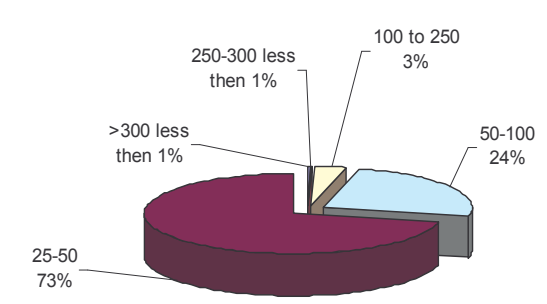

\section{Figure 16 Contamination size distribution}

The presence of humans on board determines waste water composition and existence of different types of hydrocarbons including long chain alcohols, which act as effective surfactants. Figure 17 shows ISS waste water composition [13].

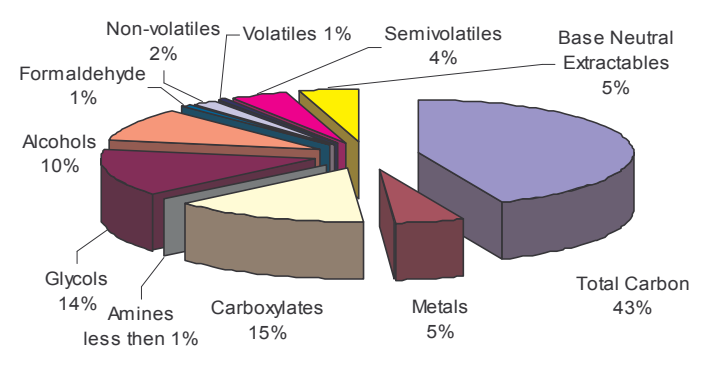

\section{Figure 17 Waste water composition}

Water samples were collected through several ISS expeditions and analyzed by US and Russian specialists.

Space Shuttle waste water will contain more organics in it than ISS condensate based on the design of the system. We believe that this will significantly slow sublimation of Space Shuttle waste water particles. At this moment we cannot definitely say by how much the process of sublimation will be retarded.

\section{CONCLUSIONS}

As a result of this study we were able to show that particle survival in LEO primarily depends on the density of the atmosphere at the time of the vent. In most cases of average or high atmosphere particles will reach safe altitudes before they sublimate to safe sizes. Uncertainties in atmospheric density, bubble inclusion, and the presence of surfactants all add to a large uncertainty band when forecasting exact trajectories for a family of candidate drop sizes in the vented plume. In the worst case scenario of low atmosphere and cold environment, the largest particles will sublimate to sub-millimeter sizes within 2.5 days, thus presenting a potential threat to lower-orbiting spacecraft. Based on the results of this study and to mitigate the risk of ice 
particles from ISS waste water damaging Shuttle tiles we developed a flight rule that restricts any waste water vents by ISS three days prior to any Shuttle launch. We are in the process of refining the analytic tools to narrow the predicted range of altitudes and phase angles of the vented stream of particles, such that planned ventings can be timed to preclude intersection of the vented particles with any high-value spacecraft in crossing orbits below the vent. A new class of ballistic forecasting tool must be developed to accomplish this, accepting dramatically carrying ballistic number and rapid altitude decay in a precise density profile that varies with location, altitude, and daily solar and geomagnetic parameters.

The effect of surfactants on sublimation of ice particles should be studied further to evaluate the effect on sublimation rates and reduce the potential hazard of catastrophic event to a lower flying vehicle.

\section{ACKNOWLEDGMENTS}

The authors are grateful for all information that was acquired by phone conversations and via email with Dr. Eugene Ungar, Johnson Space Center; Dr. Brian Swanson, University of Washington; Dr. Michael Kosch, Lancaster University; Dr. Raul Baragiola, Virginia University; Dr. Josette Bellan, Jet Propulsion Lab.

\section{REFERENCES}

1. Phenomenology of a Water Venting in Low Earth Orbit. Kofsky et all. Acta Astronautica Vol 26. No 5 pp. 325-347, 1992

2. Characteristics, Control and Uses of Liquid Streams in Space, E.P. Muntz, Meliisa Orme, AIAA Journal, May 1987, pp. 746-756

3. Scattering properties of ice particles formed by release of $\mathrm{H}_{2} \mathrm{O}$ in Vacuum. Tomas $\mathrm{T}$. Kassal, Journal of Spacecraft and Rockets V 11, pp. 54-56, 1974

4. Radiation in the Atmosphere. Kondratyev K.Ya. International Geophysics Series. Volume 12. 1969.

5. Absorption and scattering of light by small particles. Craig F. Bohren, Donald R. Huffman. John Wiley \& Sons, 1998

6. Scattering of Radiation by a Large Particle with a Random Rough Surface., S. Mukai, T. Mukai, R.H. Giese, K. Weiss, R. H. Zerull. Kluwer Academic Publishers. 1981

7. http://wine1.sb.fsu.edu/chm1045/notes/Forces /Phase/Forces06.htm

8. Sublimation of ice particles from rocket exhausts in the upper atmosphere. Y. V.
Platov, M. J. Kosch, Journal of geophysical research, Vol. 108

9. The size dependence of sublimation rates for interplanetary ice particles. H. Patashnick, G. Ruprecht, The Astrophysical Journal, 197, L79-L82, 1975

10. Evaporation of Liquid Droplets Containing Surface Impurities. Y.P. Shih, D.R. Coughanowr, AIChE Journal, Vol 14, N3, 1968, pp. 502-505

11. Retardation of Water Drop Evaporation with Monomolecular Surface Films. W.D. Garret, Journal of Atmospheric Sciences, 1971, Vol 28, pp. 816-819

12. The stabilization of water mists by insoluble monolayers. H.S. Eisner, B.W. Quince, C. Slack, Disc. Faraday Soc., 30, 1960, pp. 86-95

13. Condensate Data Summary. Engineering Directorate, Water Evaluation Team, Johnson Space Center, NASA. 2005. 


\title{
IAC-06-B6.2.08
}

\section{GENERALIZED ORBITAL PROJECTIONS OF A SUBLIMATING ICE PARTICLE}

\author{
Evgeny Menkin, Ph.D., \\ ARES Corporation, Houston, TX USA \\ evgeny.menkin-1@nasa.gov
}

Jack Bacon Ph.D. P.E.

NASA Johnson Space Center, Houston TX USA

john.bacon-1@nasa.gov 


\section{Problem statement}

- In vacuum large diameter streams with high vapor pressures have a tendency to burst, forming an uncontrolled cloud of particles which subsequently freeze and eventually sublimate

- The behavior of the stream and the formation of the particles in vacuum depend on several factors such as vapor pressure, viscosity of the vented fluid, dissolved gas content, surface tension, surfactants and the density

- In this study we attempted to capture the behavior of the particle in the Low Earth Orbit, its sublimation and deorbit process 


\section{Water vent movies}




\section{Particle formation}
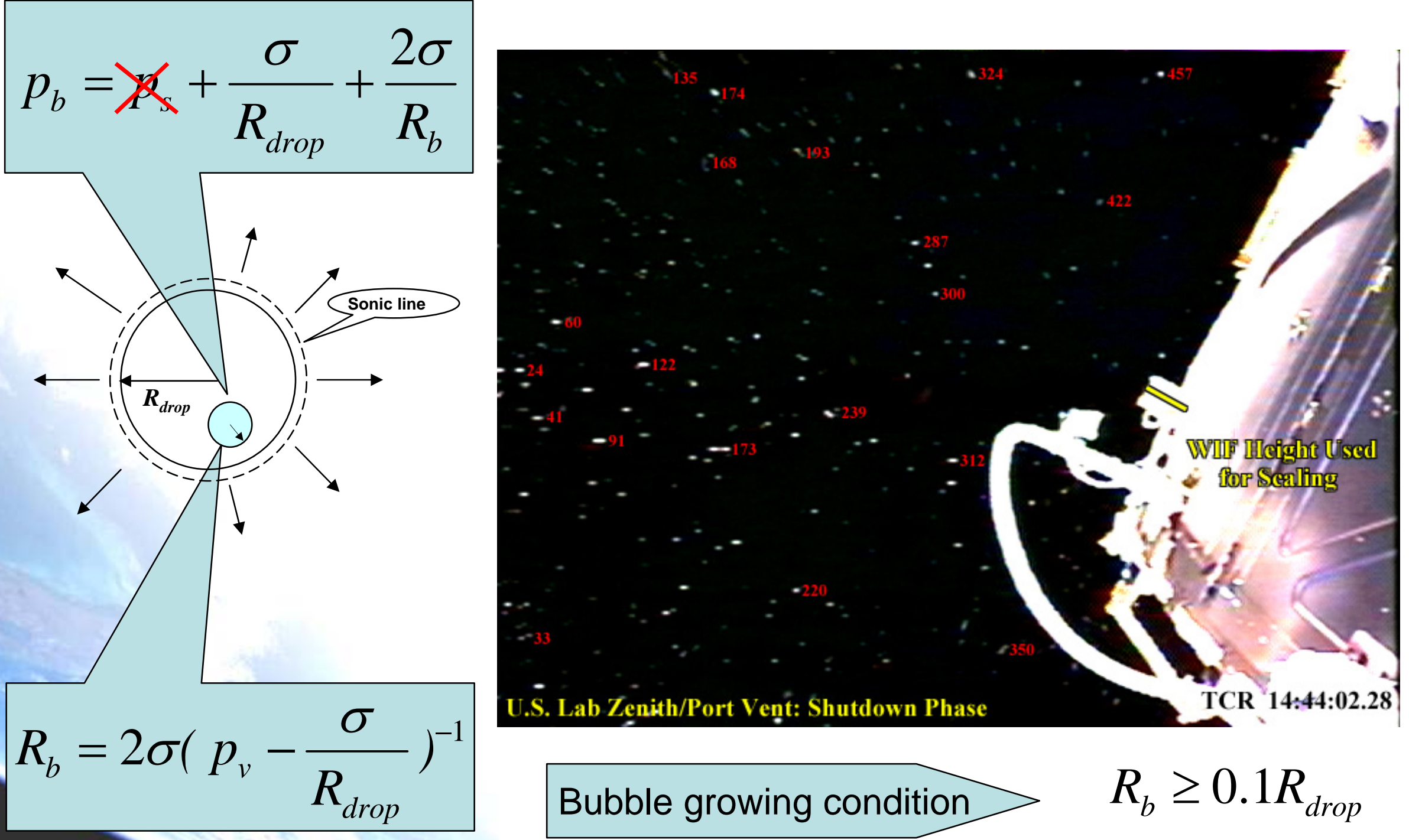

4

Bubble growing condition

$$
R_{b} \geq 0.1 R_{\text {drop }}
$$




\section{Particle model}

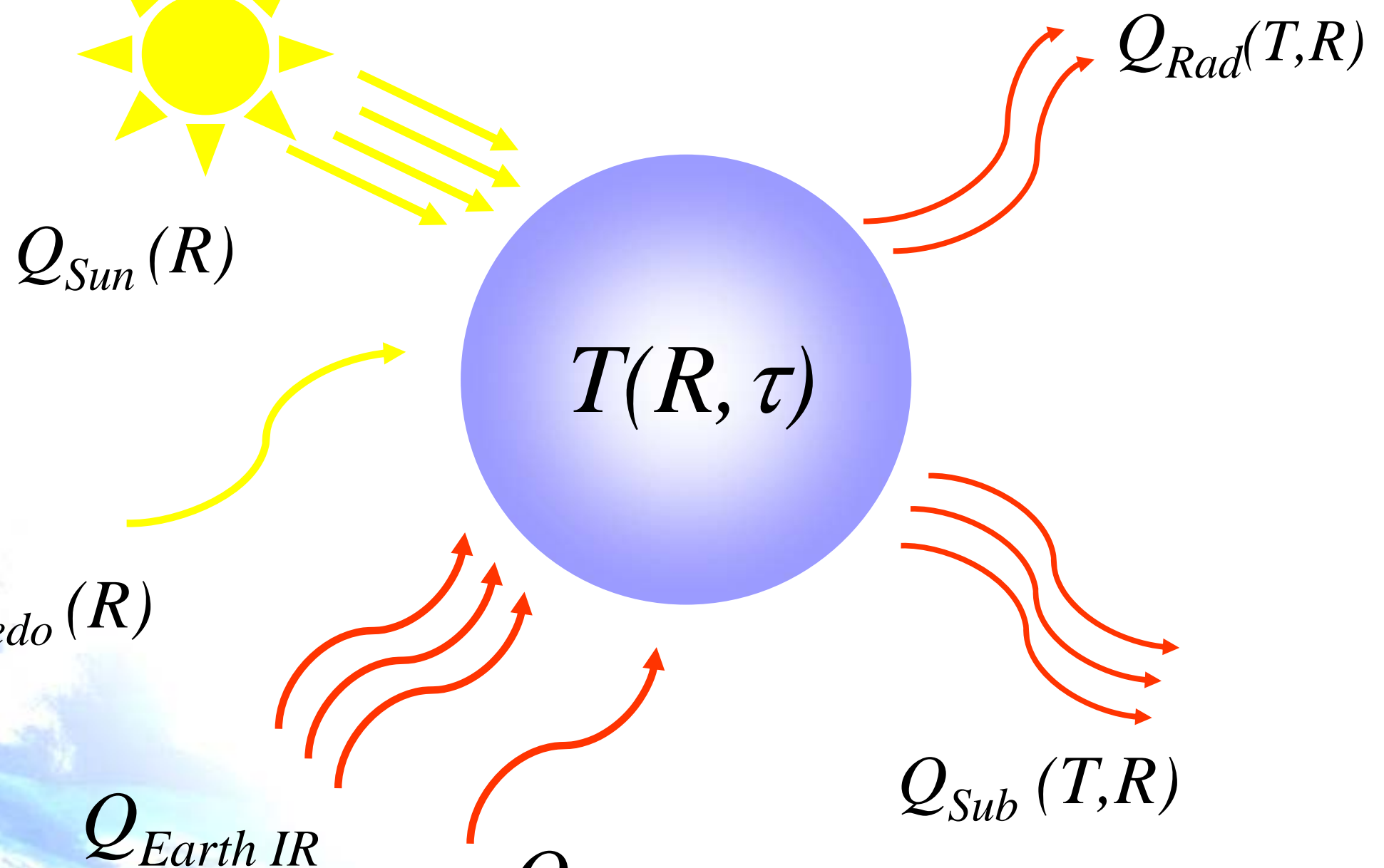

$Q_{\text {Albedo }}(R)$

$Q_{\text {Atm.heat }}$ 
- Particle Model

\section{Assumptions}

- Spherical crystalline clear ice particle

- No impurities or organic solvents.

- Clear surface with no roughening

- Homogeneous with no dissolved bubbles

- Background temperature for Earth $255 \mathrm{~K}$ at $50 \mathrm{~km}$ reference altitude

- Background temperature of Space 3K.

- Heat flux absorption by the ice particle was calculated from complex indices of refraction

$>$ Earth IR $=0.93$

$>$ Direct Sun flux $=0.27$ to 0.06

$>$ Earth albedo $=0.3$

- Atmospheric model

- Particles remain in circular or nearly circular orbit

- Initial venting altitude $=400 \mathrm{~km}$.

- The coefficient of drag $=2$, characteristic of very smooth spherical particles. 


\section{Short wave radiation absorption}

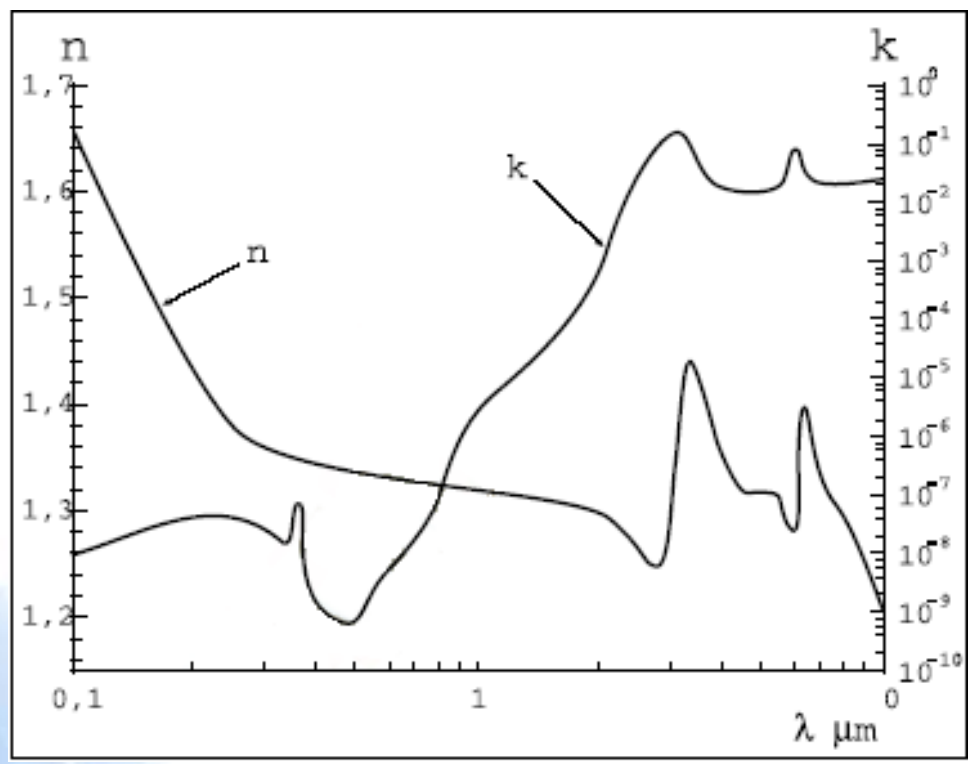

$$
\chi(R, \lambda)=\operatorname{Re}\left\{\frac{i 8 \pi R\left(m^{2}-1\right)}{\lambda\left(m^{2}+2\right)}\right\}
$$

$$
m=n-i k
$$

$Q_{\text {Sunreal }}=Q_{\text {Sunaverage }}\left(1+0.034 \cos \left(\frac{360 N}{365.25}\right)\right.$

$$
Q_{\text {Sunaverage }}=1363 \frac{\text { Watts }}{\mathrm{m}^{2}}
$$

$Q_{\text {Albedo }}=0.3 \chi\left(R_{\text {drop }}, \lambda\right) Q_{\text {sunreal }}$

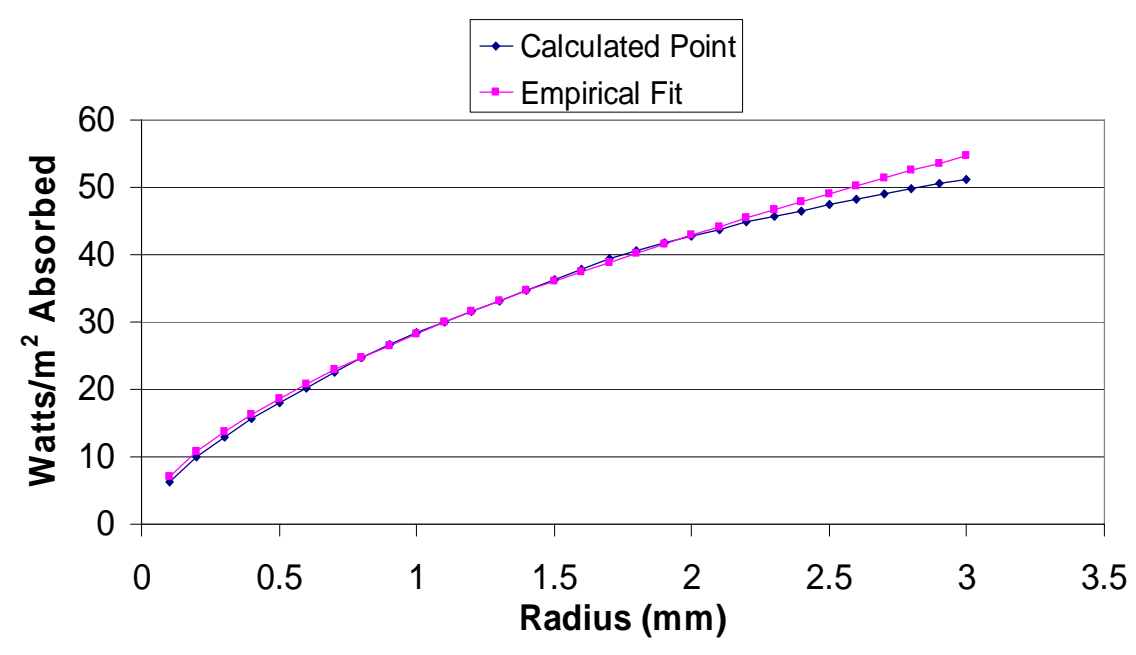




\section{Long wave radiation}

\section{Absorption}

$Q_{\text {Earth }}=4 \pi \chi\left(R_{\text {drop }}, \lambda\right) \sigma_{B} R_{\text {drop }}^{2}\left(T_{\text {drop }}^{4}-T_{\text {Earth }}^{4}\right) \varphi_{\text {Earth }}$

$\varphi_{\text {Earth }}=\frac{1-\sqrt{1-\left(\frac{R_{\text {Earth }}+50}{R_{\text {Earth }}+\text { Alt }}\right)^{2}}}{2}$

$\chi\left(R_{\text {drop }}, \lambda\right)=0.93$

\section{Emissivity}

$$
\begin{gathered}
\varphi_{\text {Space }}=1-\varphi_{\text {Earth }} \\
\varepsilon=0.96
\end{gathered}
$$

Black Body Emission Curves of the Sun and Earth

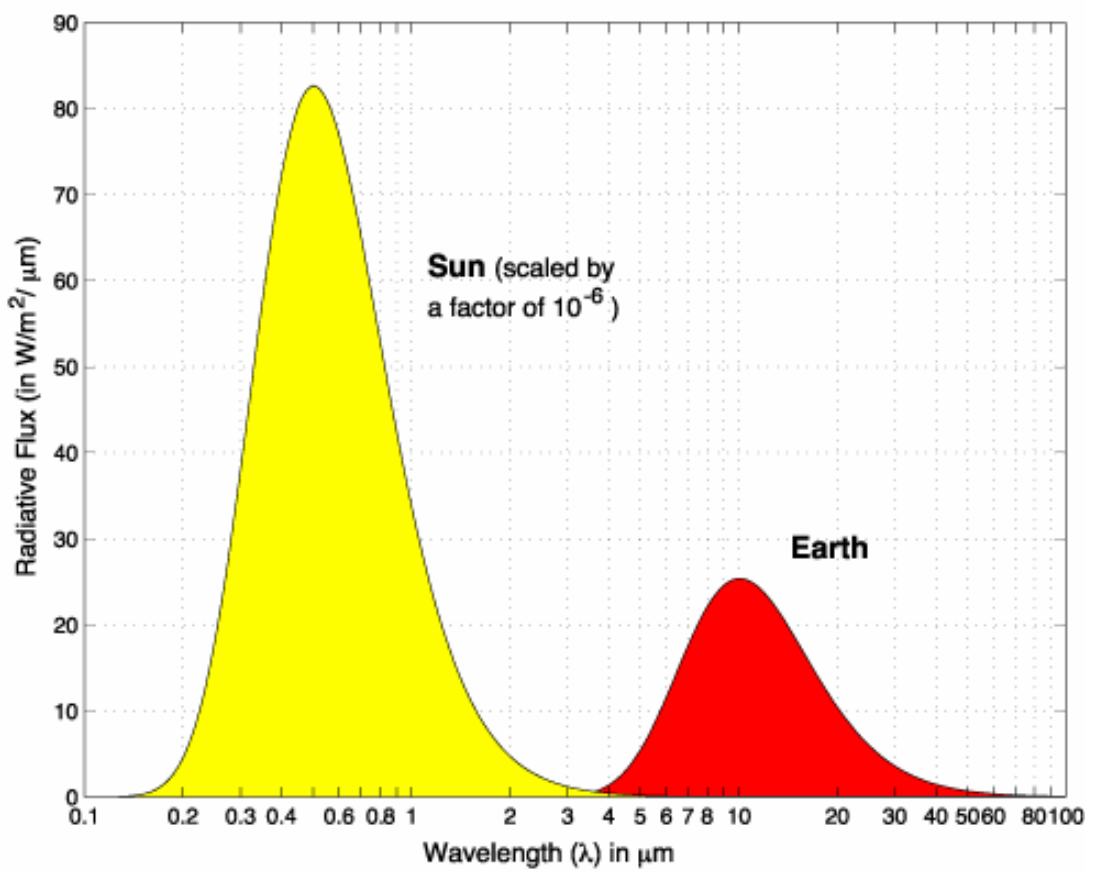

$$
Q_{\text {Space }}=4 \pi \varepsilon \sigma_{B} R_{\text {drop }}^{2}\left(T_{\text {drop }}^{4}-T_{\text {Space }}^{4}\right) \underline{\underline{\varphi_{\text {Space }}}}
$$




\section{Particle decay rate}

$\frac{d V}{d \tau}=\frac{3}{8} \frac{C_{D}}{\rho_{\text {drop }} R_{d r o p}} \rho_{A t m} V^{2} \quad C_{D}=2 \quad$ For smooth spheres

$$
V=\sqrt{\frac{\mu}{\left(R_{\text {Earth }}+A l t\right)}}
$$

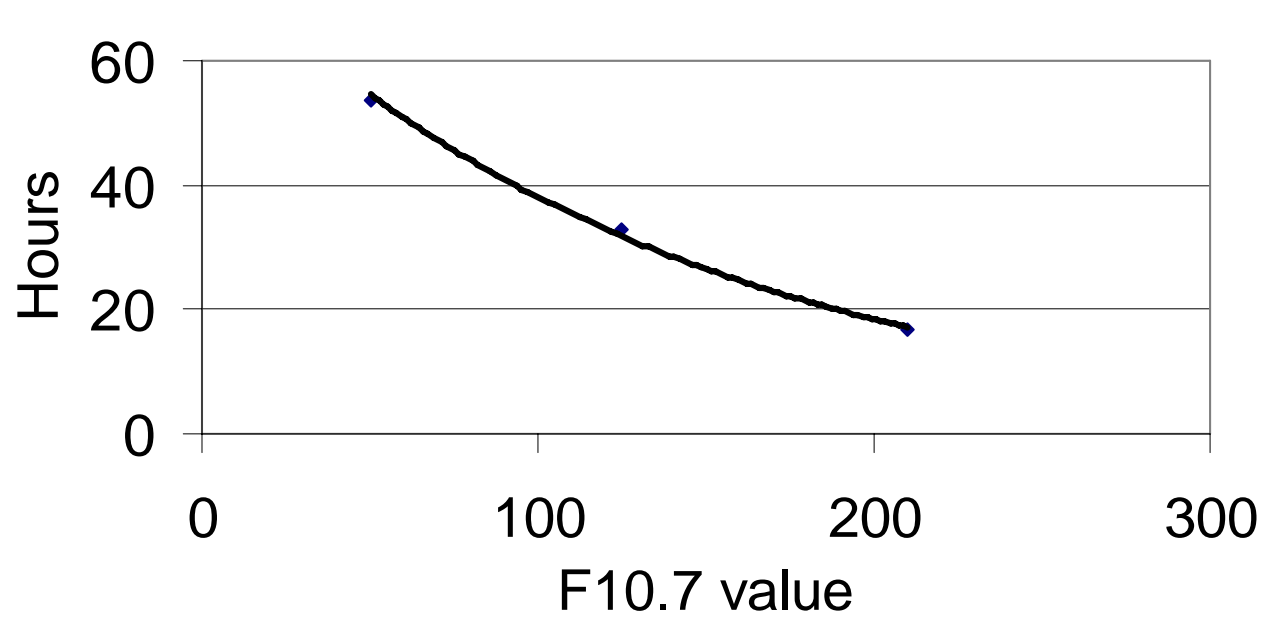

$$
\frac{d\left(A l t+R_{\text {Earth }}\right)}{\left(A l t+R_{\text {Earth }}\right)}=2 \frac{d V}{V}
$$

$$
\frac{d A l t}{d \tau}=\frac{3}{2} \frac{C_{D}}{\rho_{\text {drop }} R_{\text {drop }}} \sqrt{\frac{\rho_{\text {Atm }}(\mu)}{\left(A l t+R_{\text {Earth }}\right)}}
$$




\section{Analysis}
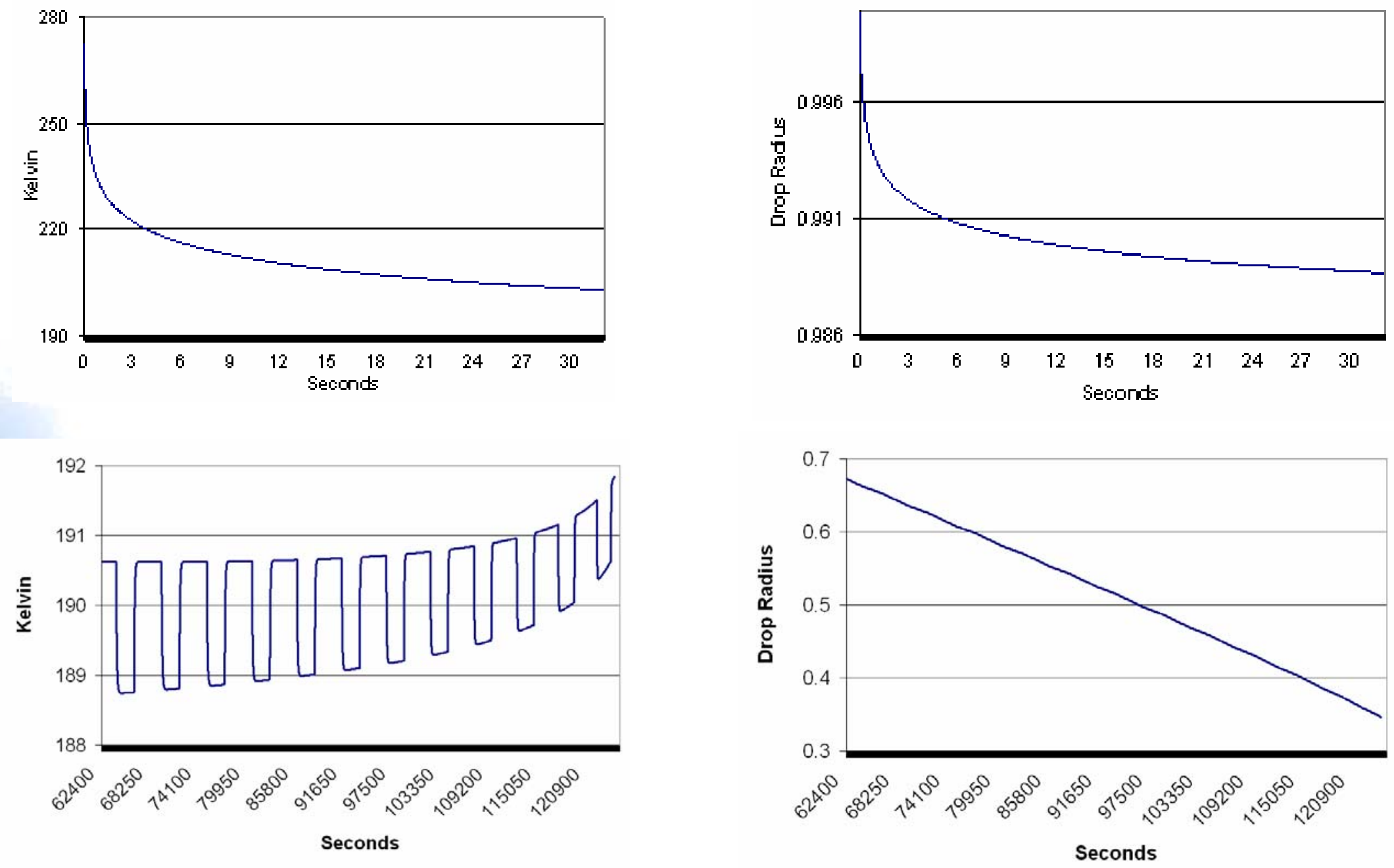


\section{Analysis}
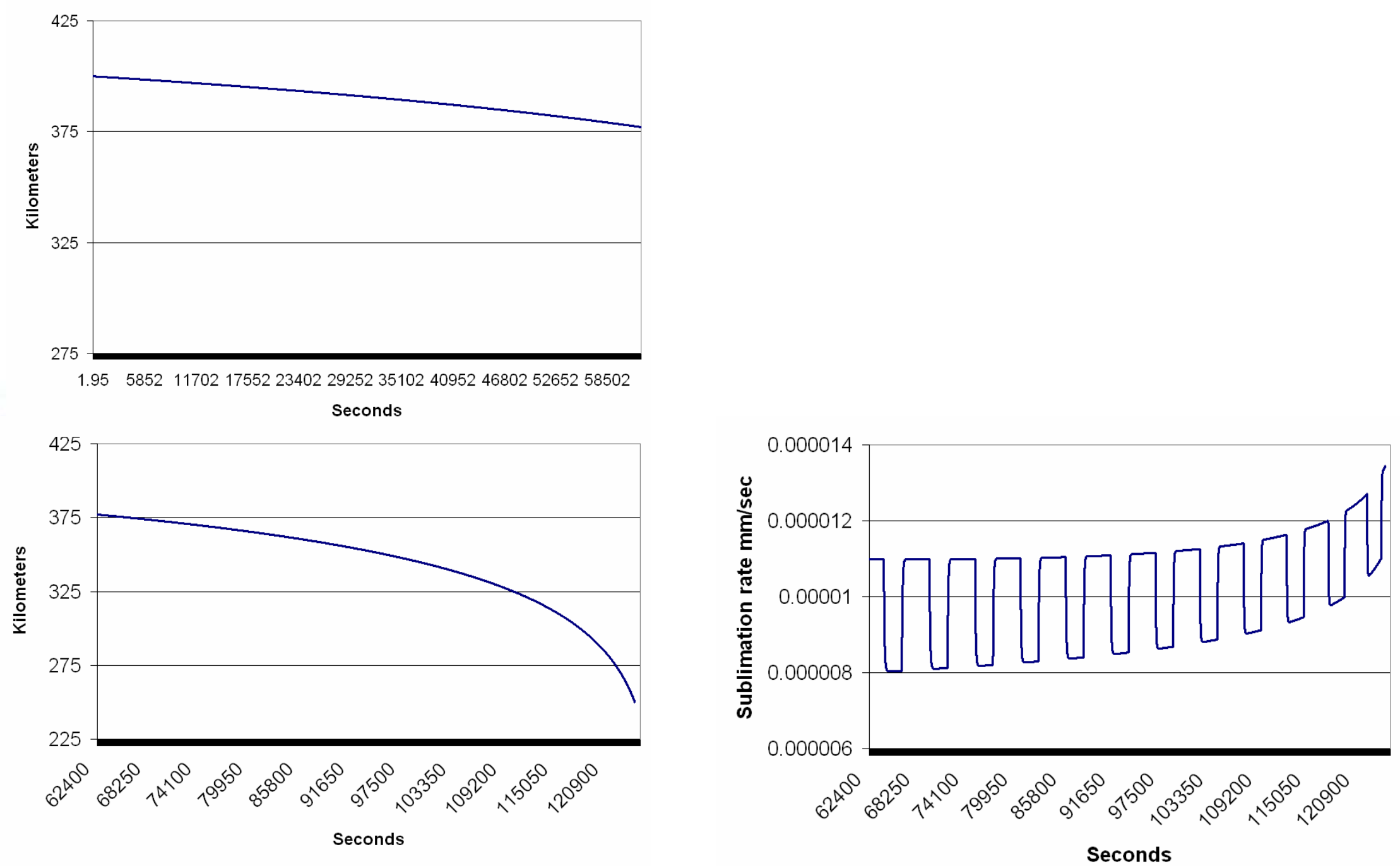


\section{Conclusions}

Particle survival in LEO primarily depends on the density of the atmosphere at the time of the vent

Uncertainties in atmospheric density, bubble inclusion, and the presence of surfactants all add to a large uncertainty band when forecasting exact trajectories for a family of candidate drop sizes in the vented plume.

Based on the results of this study we developed a flight rule that restricts any waste water vents by ISS three days prior to any Shuttle launch

We are in the process of refining the analytic tools to narrow the predicted range of altitudes and phase angles of the vented stream of particles to preclude intersection of the vented particles with any spacecraft in crossing orbits below the vent

The effect of surfactants on sublimation of ice particles should be studied further to evaluate the effect on sublimation rates and reduce the potential hazard of catastrophic event to a lower flying vehicle 